\title{
Effect of nonlinear wave-current interaction on flow fields and hydrodynamic forces*
}

\author{
WANG Tao (王 涛) and LI Jiachun (李家春) \\ (Institute of Mechanics, Chinese Academy of Sciences, Beijing 100080, China)
}

Received November 20, 1996

\begin{abstract}
A fifth-order theory for solving the problem of interaction between Stokes waves and exponential profile currents is proposed. The calculated flow fields are compared with measurements. Then the errors caused by the linear superposition method and approximate theory are discussed. It is found that the total wave-current field consists of pure wave, pure current and interaction components. The shear current not only directly changes the flow field, but also indirectly does so by changing the wave parameters due to wave-current interaction. The present theory can predict the wave kinematics on shear currents satisfactorily. The linear superposition method may give rise to more than $40 \%$ loading error in extreme conditions. When the apparent wave period is used and the Wheeler stretching method is adopted to extrapolate the current, application of the approximate theory is the best.
\end{abstract}

Keywords: Stokes wave, exponential current, nonlinear interaction.

A combination of wave and current forces acting on the submerged part of an offshore platform is the major environmental loading. So the problem of wave-current interaction has attracted the interest of a large number of researchers. We are now involved with two classes of problems: the variation of wave parameters in the process when a wave encounters a current and their interaction in the steady wave-current coexisting field. We have already investigated the former before $^{[1]}$. In this paper, we emphasize the analysis of the interaction in the coexisting field.

The water particle velocity in the wave-current field is usually predicted by using the linear superposition method; that is, the current is simply added to the wave-induced velocity in quiescent water condition. As a current is only defined to the still water level, the current profile between the still level and the wave crest has to be assumed. Bea ${ }^{[2]}$ reported that the conventional method was too crude; namely, it might be conservative when the current was flowing in the direction of wave propagation, and might be risky for the current travelling against the wave. Hence, wave-current interaction cannot be neglected. To meet the need of engineering design, various approximate methods based on the linear superposition principle have been suggested to take the effect of interaction into consideration by making appropriate modifications in wave period or current profile ${ }^{[3]}$. However, we are still not sure in what circumstance they will underestimate or overestimate the forces and how large the errors will be. Therefore, it is necessary to make clear the influence of wave-current interaction on design loading and the applicability of the approximate methods ${ }^{[4,5]}$.

Peregrine $^{[6]}$, Srokosz ${ }^{[7]}$ and Toumazis and Ahilan ${ }^{[3]}$ have reviewed the studies in this area, mentioning both small and finite amplitude models. Besides the straightforward solution for 
uniform current, the analytic solution for Stokes waves on linear or bilinear currents has been developed. Cummims and Swan ${ }^{[8]}$ extended the bilinear model to a five layer description of the flow field to represent strongly sheared currents with reduced discontinuity in vorticity, but the convergence of the solution seemed difficult to achieve. Skyner and Easson ${ }^{[9]}$ measured the velocity profiles for steep, steady waves travelling on shear currents. With the help of symbolic computation, we have found the solution of Stokes waves with the coexistence of shear flows with exponentially decaying profile in the vertical direction. The corresponding flow fields are compared with the experimental results of Skyner. And the effect of nonlinear wave-current interaction on wave kinematic and hydrodynamic forces is analyzed. Then the approximate methods are evaluated.

\section{Analytic theory}

\subsection{Statement of the problem}

We consider the problem of wave motion in vertically varying currents. The velocities of general circulations and currents due to tides or storm surges are at most of the order of a few knots, which are much smaller than the phase velocity of wave propagation. Hence, the current of practical interest can be considered moderately weak. Since the time scale of current is mostly much longer than the period of gravity wave, steady current is believed to be a reasonable assumption. The problem is formulated in a reference frame moving along with the wave, so that the flow looks stationary in this frame. However, except for uniform flow and linear shear cases, the irrotational assumption is no longer applicable due to velocity shear. Instead, the Dean's stream function formulation can be applied; namely, the governing equation becomes

$$
\nabla^{2} \psi=-\Omega(\psi) \text {, }
$$

where $\psi$ is the stream function, $\Omega$ is the vorticity.

Most studies available directly solved eq. (1) to derive the analytic solutions, in which both the stream function and the unknown free surface needed expanding. To eliminate the difficulties lying in the unknown free surface, Dubreil-Jacotin developed a coordinate transformation which maps the wave domain into a rectangle and fixes the free boundary at $\psi=0$ (see fig. 1) by regarding $y$ as a function of $x$ and $\psi, y=y(x, \psi)$.

It is convenient to further non-dimensionalize the equation based on the wave number $k$ and the phase velocity $C$. Then the resulting nonlinear equation is

$$
y_{x x} y_{\psi}^{2}-2 y_{x} y_{\psi} y_{x \psi}+\left(1+y_{x}^{2}\right) y_{\psi \psi}=\Omega(\psi) y_{\psi}^{3} \text {. }
$$

Despite the obviously more complicated governing equation, this is a small price to pay for simplifying the boundary condition. Moreover, the behaviour of the perturbation series in large wave amplitude situation is better than that of otherwise obtained series ${ }^{[10,11]}$. Applying eq. (2), $\mathrm{Hui}^{[10]}$ developed an analytic solution for pure wave, and Chaplin ${ }^{[12]}$ conducted a numerical simulation for wave on arbitrary current profile.

At the free surface $\psi=0$, the non-dimensional Bernoulli integral with constant pressure can be written as

$$
\frac{1}{2} \frac{1+y_{x}^{2}}{y_{\psi}^{2}}+\delta y=\text { constant }, \psi=0
$$



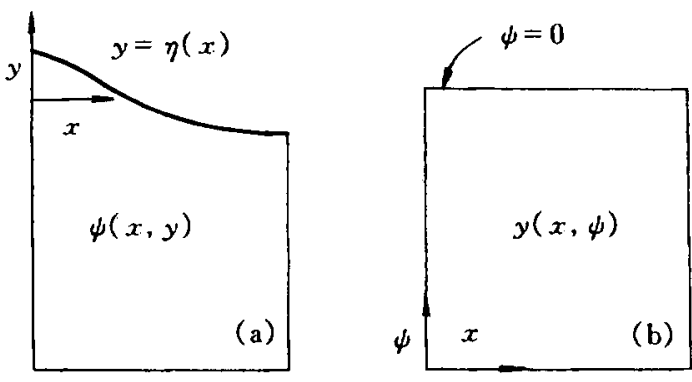

Fig. 1. Reference frame. (a) Cartesian; (b) DubreilJacotin where $\delta=\left(C_{0} / C\right)^{2}, C_{0}=\sqrt{g / k}, C_{0}$ is the phase speed of infinitesimal wave and $g$ is the gravity acceleration. Finally, the bottom boundary condition reads

$$
y \rightarrow \psi, \psi \rightarrow-\infty .
$$

\subsection{Perturbation solution}

The solution is expanded in a double series of parameters $h$ and $\varepsilon$, where $h$ is the amplitude of the first harmonic wave scaled by $k^{-1}$ and $\varepsilon$ is the

vorticity strength scaled by $C k$. Since the current is moderately weak, $h$ and $\varepsilon$ are assumed to be of the same order. Then the whole solution is regarded as the sum of uniform flow, pure wave, pure current and interaction terms:

$$
y(x, \psi)=\psi+w(x, \psi)+c(x, \psi)+n(x, \psi),
$$

where $w(x, \psi), c(x, \psi)$ and $n(x, \psi)$ are three series depending on $h$ alone, $\varepsilon$ alone and both $h$ and $\varepsilon$ respectively:

$$
\left\{\begin{array}{l}
w(x, \psi)=\sum_{i=1}^{5} w_{i}(x, \psi) h^{i}, \\
c(x, \psi)=\sum_{i=1}^{5} c_{i}(x, \psi) \varepsilon^{i}, \\
n(x, \psi)=\sum_{i, j \geqslant 1, i+j \leqslant 5} n_{i j}(x, \psi) h^{i} \varepsilon^{j} .
\end{array}\right.
$$

Eq. (5) is reduced to pure wave or pure current case as $\varepsilon$ or $h$ tends to be zero. Evidently, the isolation of the interaction term $n(x, \psi)$ facilitates the analysis of the effect of wave-current interaction, and the results for pure wave and pure current given by $\mathrm{Hui}^{[10]}$ and $\mathrm{Kirby}^{[13]}$ respectively can be directly made use of.

In the case of pure current, $c(x, \psi)$ is independent of $x$ since the considered current profile varies only in the vertical direction. The governing equation can be greatly simplified:

$$
c_{\psi \psi}=\Omega(\psi) c_{\psi}^{3} \text {. }
$$

We consider a shear current with exponentially decaying profile in the vertical direction $\Omega=\varepsilon e^{\psi}$, which is the same as indicated by Phillips ${ }^{[14]}$. By perturbation methods, the solution is easily shown to be

$$
c(\psi)=\psi+\varepsilon \mathrm{e}^{\psi}+\frac{3}{4} \varepsilon^{2} \mathrm{e}^{2 \psi}+\frac{5}{6} \varepsilon^{3} \mathrm{e}^{3 \psi}+\frac{35}{32} \varepsilon^{4} \mathrm{e}^{4 \psi}+\frac{63}{40} \varepsilon^{5} \mathrm{e}^{5 \psi} .
$$

The current profile can be expressed in Cartesian coordinate system as

$$
U=-\varepsilon \mathrm{e}^{y}\left(1-\frac{1}{2} \varepsilon \mathrm{e}^{y}+\frac{1}{4} \varepsilon^{2} \mathrm{e}^{2 y}-\frac{1}{8} \varepsilon^{3} \mathrm{e}^{3 y}+\frac{1}{16} \varepsilon^{4} \mathrm{e}^{4 y}\right),
$$

which is closer to field measurements than the uniform and linear ones ${ }^{[3]}$. It should be pointed out that the wave is travelling to the left; negative $U$ (negative $\varepsilon$ ) represents following current, while positive $U$ (positive $\epsilon$ ) represents opposing current .

To seek a periodic solution satisfying the bottom boundary condition, it is reasonable to as- 
sume the form of the interaction function $n_{i j}(x, \psi)$ as the product of triangular functions of $x$ and exponential functions of $\psi$. With the help of the symbolic computation software MATHEMATICA, the interaction function can be found by using the surface boundary condition since the governing equation is automatically satisfied. The lengthy coefficients have been shown in ref. [4]. Now we lay emphasis on analyzing the wave parameters and flow fields when wave and current are coexisting.

The wave height $H$ in current can be directly obtained from eq. (5) by evaluating it at $\psi=0$,

$$
H=2 h+\frac{10}{3} h \varepsilon+\frac{3}{4} h^{3}+\frac{153}{28} h^{3} \varepsilon+\frac{73}{24} h^{5}+\frac{3593}{196} h^{3} \varepsilon^{2} .
$$

The dispersion relationship is also determined by the boundary condition

$$
\begin{aligned}
\frac{C}{C_{0}}= & 1+\frac{2}{3} \varepsilon+\frac{1}{2} h^{2}+\frac{8}{9} \varepsilon^{2}+\frac{38}{15} h^{2} \varepsilon+\frac{37}{27} \varepsilon^{3}+\frac{1}{2} h^{4}+\frac{8153}{1350} h^{2} \varepsilon^{2}+\frac{61}{27} \varepsilon^{4} \\
& +\frac{19}{5} h^{4} \varepsilon+\frac{603}{50} h^{2} \varepsilon^{3}+\frac{874}{243} \varepsilon^{5} .
\end{aligned}
$$

The above two equations show that when current is vertically sheared, the wave parameters in the coexisting field are different from the pure wave ones. The parameters depend not only on the wave amplitude $h$, but also on the vorticity strength $\varepsilon$ due to current. Generally speaking, following current tends to increase the wave height and phase velocity, and opposing current tends to decrease them. The variation of wave parameters will certainly lead to the change of flow fields.

In the Dubreil-Jacotin formulation, the horizontal and vertical velocity components can be expressed in the following form:

$$
u=\frac{1}{y_{\psi}}, v=\frac{y_{x}}{y_{\psi}} .
$$

If the inverse function $\psi=\psi(x, y)$ is found from eq. (5), it is not difficult to calculate the velocity and corresponding acceleration fields in the Cartesian coordinate system. The results are transformed to the physical plane by removing the uniform flow part due to the moving reference frame. Since the maximum horizontal force and capsizing moment are of the most importance in ocean engineering, the horizontal velocity component $u$ is investigated in detail. The results show that the total flow field in a wave-current environment consists of pure wave $u_{\mathrm{w}}$, pure current $U$ and interaction component $u_{\mathrm{n}}$,

$$
u=u_{\mathrm{w}}+U+u_{\mathrm{n}},
$$

where $u_{\mathrm{w}}$ and $U$ are the same as the pure wave and pure current profiles. They are series of $h$ and $\varepsilon$ respectively. The interaction term $u_{\mathrm{n}}$ can be written as

$$
u_{\mathrm{n}}=\sum_{i, j \geqslant 1, i+j \leqslant s} m_{i j} h^{i} \varepsilon^{j} .
$$

The coefficient $m_{i j}$ is shown as the product of three matrixes $S, M_{i j}$ and $E, m_{i j}=S M_{i j} E$, where

$$
\begin{aligned}
& S=\left\{\begin{array}{lll}
\left(\begin{array}{ccc}
\cos x & \cos 3 x & \cos 5 x
\end{array}\right), & \text { if } i \text { is odd; } \\
\left(\begin{array}{lll}
1 & \cos 2 x & \cos 4 x
\end{array}\right), & \text { if } i \text { is even. }
\end{array}\right. \\
& E=\left(\mathrm{e}^{\psi} \mathrm{e}^{2 \psi} \mathrm{e}^{3 \psi} \mathrm{e}^{4 \psi} \mathrm{e}^{5 \psi}\right)^{\mathrm{T}},
\end{aligned}
$$




$$
\begin{aligned}
& M_{11}=\left(\begin{array}{ccccc}
0 & -\frac{4}{3} & 0 & 0 & 0 \\
0 & 0 & 0 & 0 & 0 \\
0 & 0 & 0 & 0 & 0
\end{array}\right) \\
& M_{13}=\left(\begin{array}{ccccc}
\frac{7}{18} & \frac{35}{9} & 0 & -\frac{7}{2} & 0 \\
0 & 0 & 0 & 0 & 0 \\
0 & 0 & 0 & 0 & 0
\end{array}\right) \text {, } \\
& M_{21}=\left(\begin{array}{ccccc}
0 & 0 & -\frac{3}{2} & 0 & 0 \\
0 & -\frac{4}{5} & -\frac{47}{30} & 0 & 0 \\
0 & 0 & 0 & 0 & 0
\end{array}\right) \text {, } \\
& M_{41}=\left(\begin{array}{ccccc}
0 & \frac{887}{180} & \frac{27}{8} & -\frac{6}{5} & -\frac{61}{9} \\
0 & \frac{221}{1260} & \frac{53}{40} & -\frac{272}{105} & -\frac{5741}{630} \\
0 & 0 & 0 & -\frac{3982}{2835} & -\frac{883}{378}
\end{array}\right) \text {, } \\
& M_{12}=\left(\begin{array}{ccccc}
\frac{35}{12} & 0 & -\frac{25}{12} & 0 & 0 \\
0 & 0 & 0 & 0 & 0 \\
0 & 0 & 0 & 0 & 0
\end{array}\right) \\
& M_{31}=\left(\begin{array}{ccccc}
\frac{887}{180} & \frac{3}{2} & -1 & -\frac{989}{180} & 0 \\
0 & 0 & \frac{39}{35} & -\frac{53}{28} & 0 \\
0 & 0 & 0 & 0 & 0
\end{array}\right) \text {, } \\
& M_{32}=\left(\begin{array}{ccccc}
\frac{126161}{21600} & \frac{887}{135} & \frac{803}{80} & -\frac{98}{25} & -\frac{27109}{1440} \\
0 & 0 & \frac{1273}{39200} & -\frac{638}{147} & -\frac{66001}{10080} \\
0 & 0 & 0 & 0 & 0
\end{array}\right) \text {, } \\
& M_{23}=\left(\begin{array}{ccccc}
0 & \frac{7}{18} & \frac{35}{4} & 0 & -\frac{155}{18} \\
0 & \frac{9701}{6750} & \frac{24097}{4500} & -\frac{84}{25} & -\frac{809}{90} \\
0 & 0 & 0 & 0 & 0
\end{array}\right) ; \\
& M_{22}=\left(\begin{array}{ccccc}
0 & \frac{35}{12} & 0 & -\frac{67}{18} & 0 \\
0 & \frac{359}{300} & -\frac{44}{25} & -\frac{39}{10} & 0 \\
0 & 0 & 0 & 0 & 0
\end{array}\right) \text {, } \\
& M_{14}=\left(\begin{array}{ccccc}
\frac{203}{432} & \frac{14}{27} & \frac{875}{144} & 0 & -\frac{49}{8} \\
0 & 0 & 0 & 0 & 0 \\
0 & 0 & 0 & 0 & 0
\end{array}\right) .
\end{aligned}
$$

The superscript $T$ means transposition of a matrix.

If the interaction terms equal zero, the analytic theory is reduced to the linear superposition solution. When linear superposition is applied, neither the velocity and acceleration nor the wave parameters in the flow fields can reflect the effect of nonlinear wave-current interaction. Since the perturbation parameters $h$ and $\varepsilon$ are fixed, the present theory is suitable for the condition when wave and coexisting current are travelling forward together.

\subsection{Effect of interaction on flow fields}

The distributive force $f$ exerted on small-scale offshore structures is usually predicted by Morison equation:

$$
f=\frac{1}{2} C_{\mathrm{d}} \rho D|u| u+\frac{\pi}{4} C_{\mathrm{m}} \rho D^{2} \dot{u},
$$

where $C_{d}$ and $C_{m}$ are the drag and inertia coefficients respectively, $\rho$ the water density, $D^{-}$the cylinder diameter. The total force $F$ on the cylinder is given by the integration of eq. (15). Since the drag proportional to the square of the total wave-current velocity $u$ is the dominant force acting on small-scale structures such as the legs of a jacket platform, the loading error doubles the error in particle velocity. Therefore, it is very important to accurately compute the velocity field.

Skyner measured the wave kinematics on shear currents by the method of particle image ve- 
locimetry (PIV). The main current condition was generated by introducing a flow opposed to the wave along the bottom of the flume. To show the effect of nonlinear interaction on flow fields, the velocity profiles predicted by the present theory and linear superposition method are plotted in fig. 2, along with the experimental measurements. The perturbation parameters $h$ and $\varepsilon$ are derived from eqs. (9)-(11) according to the current velocity, wave height and wave period used in the tests. The theoretical current velocity is kept equal to the measured value at the free surface. Then the horizontal velocity component can be obtained from eq. (13). When linear superposition is used, $h$ is determined by the pure wave theory; the total velocity is calculated by adding the current to the pure wave velocity.
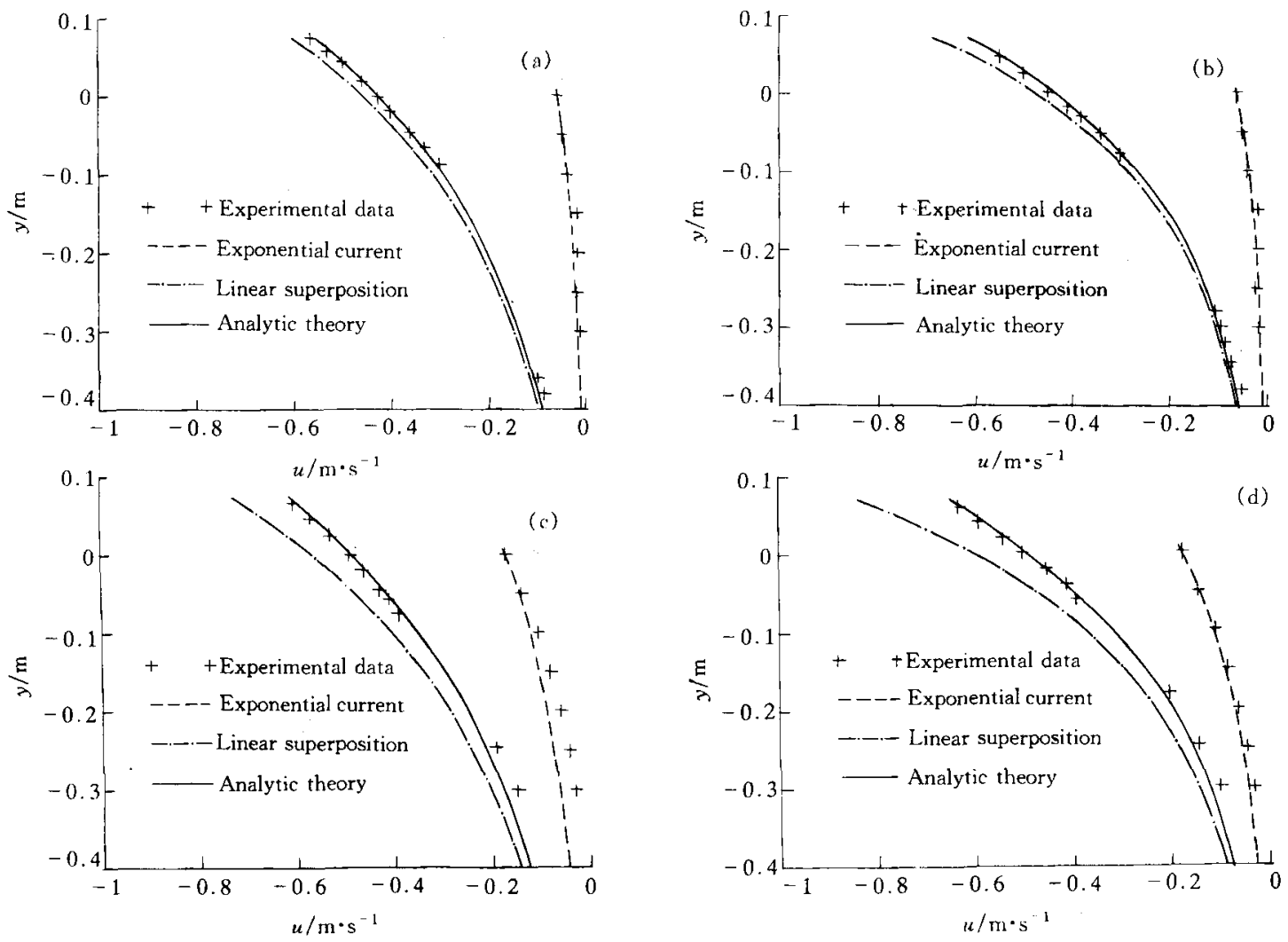

Fig. 2. Horizontal velocity under crest. (a) $U=-0.06 \mathrm{~m} / \mathrm{s}, H=0.13 \mathrm{~m}, T=0.98 \mathrm{~s}$; (b) $U=-0.06 \mathrm{~m} / \mathrm{s}$, $H=0.12 \mathrm{~m}, T=0.81 \mathrm{~s} ;$ (c) $U=-0.17 \mathrm{~m} / \mathrm{s}, H=0.13 \mathrm{~m}, T=0.98 \mathrm{~s}$; (d) $U=-0.17 \mathrm{~m} / \mathrm{s}, H=0.12 \mathrm{~m}$, $T=0.81 \mathrm{~s}$.

The exponential profile is a suitable model for the experimentally generated current. For weak current (see fig. 2(a), (b)), the exponential current is in agreement with the measured shear flow. When the current becomes strong (fig. $2(\mathrm{c}),(\mathrm{d})$ ), the theoretical current velocity is a little higher than the tested one away from the still water level. Because of the difficulty in generating shear flows, the current velocity was set to zero at the depth of $0.4 \mathrm{~m}$ although the water depth was $0.75 \mathrm{~m}$ in the experiments. That is to say, the current strongly sheared near the still water level existed only in the upper half of the water flume, while the theoretical profile we use can reasonably represent the shear current extending from the bottom to the free surface. 
Near the water surface, the combined wave-current velocity obtained by the present theory is consistent with the measurement for all the current conditions. Because of the difference in current profiles, the results show some difference deep in water for strong current. In this case, although the relative error may be large, it will not exert significant effect on loading since the absolute value of velocity is small. Consequently, the present theory can predict the wave kinematics on shear currents satisfactorily.

The figures also show that the error caused by the linear superposition method is within the range of $10 \%-30 \%$ and increases with the increase of wave amplitude and current strength. It reaches the maximum near the free surface and decreases rapidly with the depth, the reason for which is that the velocities of wave and current exponentially decay with depth; the interaction between them decays correspondingly.

\subsection{Effect of interaction on hydrodynamic forces}

The total force $F$ and capsizing moment $M$ derived from the present theory and linear superposition method are further compared in figs. 3 and 4 , so that the effect of nonlinear wave-current interaction on wave loading in extreme conditions can be elucidated. Typical design wave and current parameters are chosen as follows: wave height $13 \mathrm{~m}$, wave period $11 \mathrm{~s}$, current velocity $\pm 1.5 \mathrm{~m} / \mathrm{s}$, and the cylinder diameter is $1 \mathrm{~m}^{[3]}$.
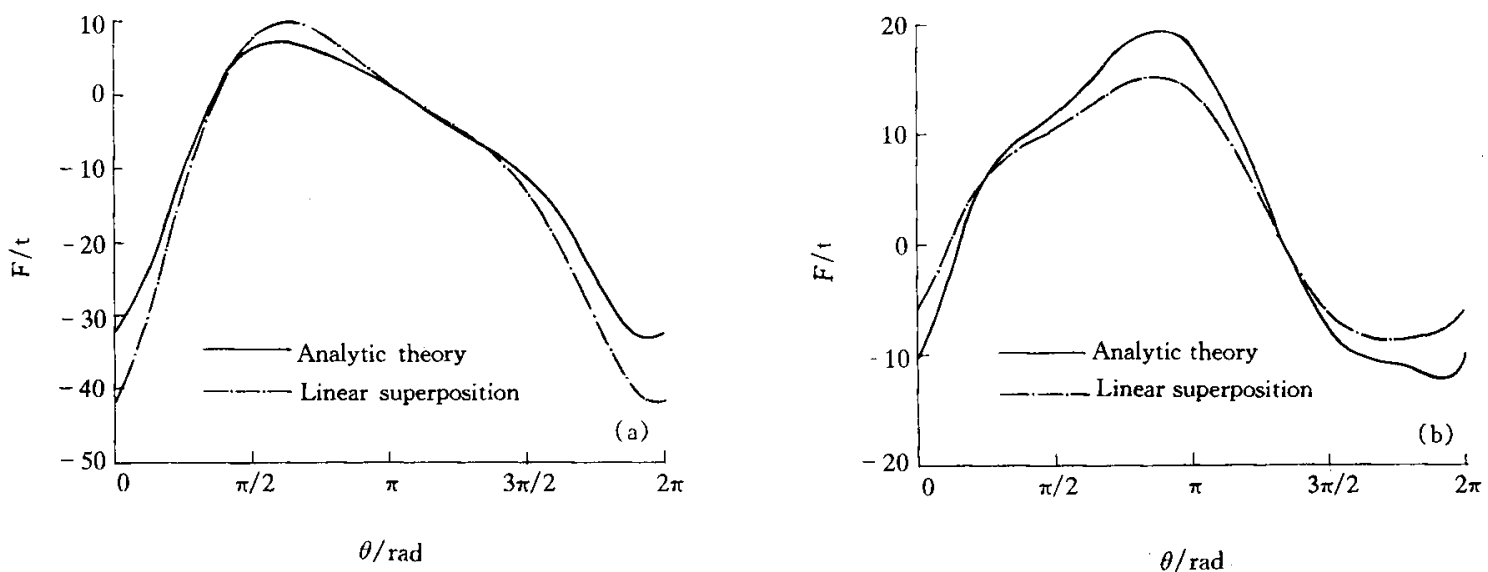

Fig. 3. (a) Total force in following current. $(U=-1.5 \mathrm{~m} / \mathrm{s}, H=13 \mathrm{~m}, T=11 \mathrm{~s})$; (b) total force in opposing current $(U=1.5 \mathrm{~m} / \mathrm{s}, H=13 \mathrm{~m}, T=11 \mathrm{~s})$.

The graphical results illustrate that the linear method overestimates the forces in following current (see figs. 3(a) and 4(a)), and underestimates them in opposing current (see figs. 3(b) and $4(\mathrm{~b})$ ). The error due to interactive effect is around $40 \%$. The qualitative conclusion is consistent with $\mathrm{Bea}^{[2]}$. As for wave of certain amplitude, the interaction of the wave with opposing current is more prominent than the interaction with following current, and the error of linear results in opposing case is $10 \%$ higher than the error in following case.

It should also be pointed out that the maximal wave-current loading does not occur exactly at but a little in front of the crest. Eq. (15) shows that the loading consists of drag and inertia components, which depend on velocity and acceleration respectively. Although the velocity is not maximal in front of the crest, the combined act of velocity and following acceleration may give the 

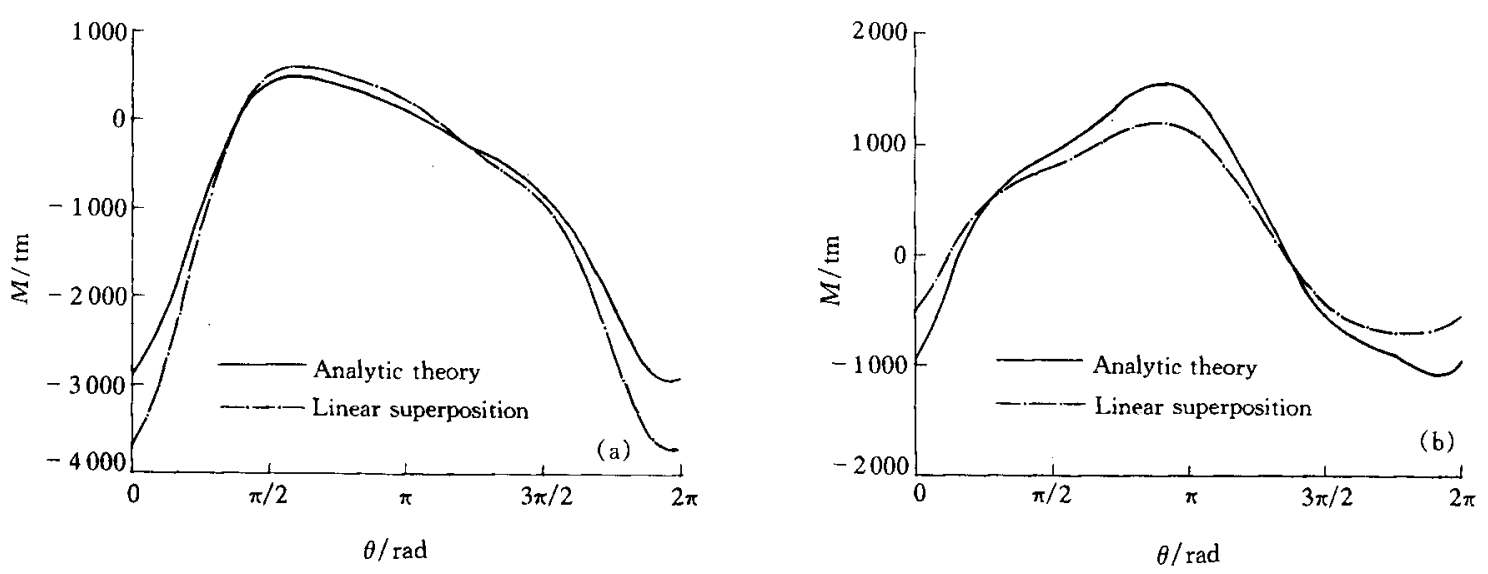

Fig. 4. (a) Capsizing moment in following current ( $U=-1.5 \mathrm{~m} / \mathrm{s}, H=13 \mathrm{~m}, T=11 \mathrm{~s})$; (b) capsizing moment in opposing current $(U=1.5 \mathrm{~m} / \mathrm{s}, H=13 \mathrm{~m}, T=11 \mathrm{~s})$.

maximum force. Since following current increases the particle velocity near the crest, the drag is much more significant than the inertia in this case, so the maximal loading appears very near the crest and decreases fast away from this phase. The effect of drag becomes relatively weak for opposing current, so the maximal loading appears somewhat far from the crest.

\section{Approximate theory}

\subsection{Review of approximate theory}

Based on the above analysis, the linear superposition method may give rise to more than $40 \%$ error in wave loading. Therefore, the effect of nonlinear wave-current interactin on flow fields and hydrodynamic forces cannot be neglected in engineering design. During these years, various modified linear superposition methods, namely the so-called approximate theory, have been proposed to approximately take account of the effect of wave-current interaction. However, these methods lack theoretical and experimental basis. It will help reduce uncertainties in wave-current loading on offshore structures to compare and evaluate these empirical approaches.

The wave velocity in a wave-current coexisting field may be obtained by using (i) actual wave period $T$; (ii) apparent wave period $T_{\text {app }}$, where $T_{\text {app }}$ is the period that an observer moving along with the depth-mean current $U_{\mathrm{m}}$ will see. Although the wave height $H$ and wave number $k$ in the moving frame of reference are the same as those in the stationary frame, the phase velocity is different:

$$
C=C_{\mathrm{r}}+U_{\mathrm{m}} \text {. }
$$

As far as the frequency is concerned, the wave frequency $\omega$ seen from the stationary frame is different from the frequency $\omega_{\mathrm{r}}$ seen from the frame moving with the current

$$
\omega=\omega_{\mathrm{r}}+k U_{\mathrm{m}} \text {. }
$$

This phenomenon is what is called the Döppler effect ${ }^{[15]}$. To keep consistency with the analytic theory, the variables are all non-dimensionalized with $C$ and $k$ in the following analysis. Since the wave appears to be propagating on still water in the moving frame, the relative frequency $\omega_{\mathrm{r}}$ can be given by linear wave theory $\omega_{\mathrm{r}}=\sqrt{g k \tanh (d)} / C k$, where $d$ is the water depth. The mean 


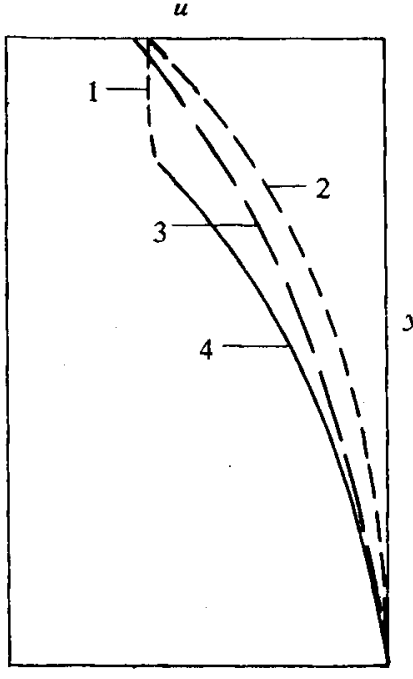

Fig. 5. Current profile extrapolation. 1, Linear adjusted; 2 , Wheeler stretching; 3 , mean position; 4 , still water.

current $U_{\mathrm{m}}$ is computed by using the first-order expression $U_{\mathrm{m}}=$ $2 / \sinh (2 d) \int_{-d}^{0} U(y) \cosh (2(y+d)) \mathrm{d} y$, derived by Kirby for arbitrary current profile $U$. With the actual wave period $T(2 \pi / \omega)$ given, eq. (17) can be solved to obtain the apparent period $T_{\text {app }}\left(2 \pi / \omega_{\mathrm{r}}\right)$.

If the current $U_{\mathrm{s}}$ is defined to the still water level, the current contribution $U$ to the total particle velocity may be obtained by a number of methods (see figure 5).

(i) Linear adjusted method ( $\mathrm{L}$ ). The current profile is extended to the crest with a uniform current equal to the value at the still water level:

$$
\left\{\begin{array}{l}
U(y)=U_{\mathrm{s}}(0), y>0 \\
U(y)=U_{\mathrm{s}}(y), y \leqslant 0 .
\end{array}\right.
$$

(ii) Wheeler stretching method $(\mathrm{W})$. The current profile is stretched vertically to the instantaneous surface $\eta$ :

$$
U(y)=U_{\mathrm{s}}\left(\frac{(y-\eta) d}{d+\eta}\right) .
$$

(iii) Mean position current (M). The local particle velocity is determined by the value at the particle mean position $y_{\mathrm{mp}}$ :

$$
U(y)=U_{\mathrm{s}}\left(y_{\mathrm{mp}}\right) .
$$

The mean position may be found using linear theory $y_{\mathrm{mp}}=y-\frac{H}{2} \frac{\sinh \left(y_{\mathrm{mp}}+d\right)}{\sinh (d)} \cos \theta$, where $H$ is the wave height, and $\theta$ is the phase angle.

There are six possible approximate methods by using different combination of wave periods and current extrapolation methods. The approximate wave-current model can be summarized in the following three steps: (i) the proper wave period is used as input in a wave model for zero current, and the particle velocity $u_{\mathrm{w}}$ due to wave is derived; (ii) the current is extrapolated to obtain the modified current velocity $U$ in the flow field; (iii) the wave induced velocity $u_{\mathrm{w}}$ is added to the current $U$; then the total water particle velocity $u$ at any depth can be calculated

$$
u(y)=u_{\mathrm{w}}(y)+U(y) .
$$

\subsection{Evaluation of approximate theory}

The distributive forces obtained by the analytic and six approximate solutions are presented in fig. 6 for the above design wave and current parameters. To show the different approximate methods in the figures, the wave contribution is represented by the actual period $T$ and the apparent period $T_{\text {app }}$; the current contribution is represented by $\mathrm{L}, \mathrm{W}$ and $\mathrm{M}$ respectively.

Generally speaking, when the actual wave period is used, the three current profile extrapolation methods cannot well model the water particle kinematics. The forces are overestimated in following current and underestimated in opposing current. The linear adjusted forces deviate signifi- 
cantly from the results of the analytic solution. If $\mathrm{W}$ or $\mathrm{M}$ is adopted, the results can be improved but they are still unsatisfactory.

The apparent wave period gives better results than the actual period for the same current profile since the Döppler effect is considered. W yields the best agreement with the analytic results and the error is restricted within $10 \%$. Physically, when wave and current are propagating in the same direction (see fig. 6 (a)), eq. (16) shows that the apparent period leads to smaller phase velocity and corresponding particle velocity than the actual period, and we can see from fig. 5 that Wheeler stretching gives the smallest current velocity in the extrapolation methods, and their combination just makes up the shortage of the linear superposition method that the wave loading is overestimated in following current. Similarly, in opposing current, their combination gives the largest forces among the approximate methods (see fig. $6(\mathrm{~b})$ ), so it is also applicable.
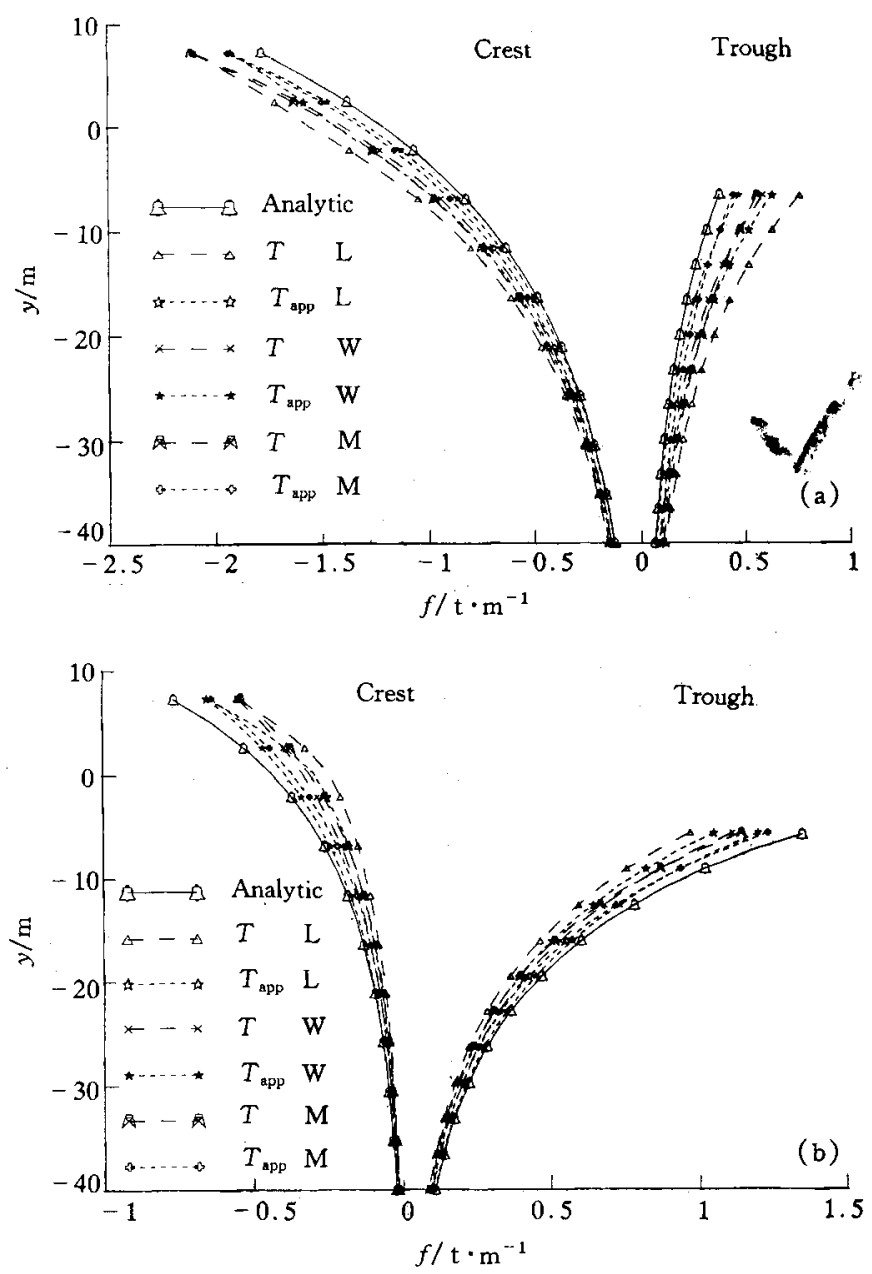

\section{Conclusions}

Fig. 6. (a) Distributive force in following current $(U=-1.5 \mathrm{~m} / \mathrm{s}$, $H=13 \mathrm{~m}, T=11 \mathrm{~s}$ ); (b) distributive force in opposing current ( $U=$

In this paper, an analytic solution $1.5 \mathrm{~m} / \mathrm{s}, H=13 \mathrm{~m}, T=11 \mathrm{~s}$ ).

for Stokes waves riding on vertically

sheared currents is presented; the effect of nonlinear wave-current interaction on flow fields and hydrodynamic forces is analyzed. The results show that wave-current interaction not only directly changes the flow fields but also alters the wave parameters. The analytic velocity is in agreement with the available measurement. The linear superposition method may cause large error in design loading. It is observed that when the Wheeler stretched current in connection with the apparent wave period is used, the wave kinematics can be generally best modeled by the approximate theory.

\section{References}

1 Wang, T., Li, J.C., Application of conservation law of wave action flux to wave-current interaction, Acta Mech. Sinica (in Chinese), 1996, 28(3):281

2 Bea, R. G., Lai, N. W., Hydrodynamic loadings on offshore platforms, in Proc. 10th Offsh. Tech. Conf., Houston; 
OTC, $1978,155$.

3 Toumazis, A.D., Ahilan, R. V., Review of recent analytical and approximate solutions of wave-current interaction, in Envir. Forces on Offsh. Struct.\& Their Predict., Netherlands: Kluwer Acad. Pub., 1990, 61.

4 Li, J.C., Chwang, T. A., Effect of,non-uniform current on wave fields, in Proc. 4 th Int. Offsh. \& Polar Eng. Conf., 1994, 3:125.

5 Osaka: ISOPE, Li, J.C., Lin, M., Wang, T., Flow interaction relevant to water waves, in Proc. 1st Int. Conf. on Flow Interact., Hong Kong: Univ. Hong Kong, 1994, 433.

6 Peregrine, D.H., Interaction of water waves and currents, Adv. in Appl. Mech ., 1976, 16:9.

7 Srokosz, M. A., Models of wave-current interaction, in Modelling the Offsh. Envir., Netherlands: Kluwer Acad. Pub., 1987,313

8 Cummims, 1., Swan, C.. Nonlinear wave current interaction, in Wave Kinemat. \& Envir. Forces, Netherlands: Kluwer Acad. Pub., 1993, 35

9 Skyner, D. J., Easson, W. J., Wave kinematics on sheared currents, in Wave Kinemat. \& Envir. Forces, Netherlands: Kluwer Acad. Pub., 1993, 101.

10 Hui, W.H., Tenti, G., A new approach to steady flows with free surfaces, J. Appl. Math. \& Phys., 1982, $33: 569$.

11 Drennan, W.M., Hui, W.H., Tenti, G., Accurate calculation of Stokes water waves of large amplitude, J. Appl. Math . \& Phys., 1992, 43:367.

12 Chaplin, J.R., Computation of steep waves on a current with strong shear near to the surface, in Water Wave Kinemat., Netherlands: Kluwer Acad. Pub., 1990, 429.

13 Kirby, J. T., Chen, T. M., Surface waves on vertically sheared flows: approximate dispersion relations, J. Geoph. Res., $1989,94: 1013$.

14 Phillips, O.M., The Dynamics of the Upper Ocean, New York: Cambridge Univ. Press, 1977, 33.

15 Gudmestad, O.T., Karunakaran, D., Wave-current interaction, in Envir. Forces on Offsh. Struch. \& Their Predict., Netherlands: Kluwer Acad. Pub., 1990, 81. 